\title{
Leitlinienprogramm
}

\section{Update der S3-Leitlinie Magenkarzinom}

\section{Unter der Federführung der Deutschen Gesellschaft für Verdauungs- und Stoffwech- selkrankheiten wurde im Leitlinienprogramm Onkologie die neue S3-Leitlinie Magen- karzinom vorgestellt. Die wichtigsten Neuerungen in der Leitlinie wurden von Prof. Markus Möhler, Universitätsmedizin Mainz, auf dem Deutschen Krebskongress vorgestellt.}

Neu in die Leitlinien aufgenommen wurde, dass Helicobacter pylori (H. pylori) der wesentliche Risikofaktor für die Entwicklung eines Magenkarzinoms ist. Daher sollte die H.-pylori-Eradikation mit dem Ziel der Magenkarzinomprävention bei Risikogruppen durchgeführt werden. Bezüglich des Screenings soll Patienten mit fortgeschrittener Atrophie und intestinaler Metaplasie des Magens eine endoskopische Überwachung angeboten werden. Ein bevölkerungsbezogenes endoskopisches Screening wird in Deutschland weiterhin nicht empfohlen.

\section{Vorsorge und Diagnostik}

Ein frühes Erkrankungsalter und das Vorliegen eines diffusen Magenkarzinoms sowie eine familiäre Häufung sind Hinweise auf ein vererbtes Magenkarzinom. In diesen Fällen sollte bei Patienten im 20. und 30. Lebensjahr eine genetische Beratung erfolgen. Bei Patienten mit hereditärem nicht polypösem kolorektalen Karzinom (HNPCC) und Risikopersonen für HNPCC sollte ab dem 35. Lebensjahr zusätzlich zur Koloskopie regelmäßig eine Ösophago-Gastro-Duodenoskopie (ÖGD) durchgeführt werden. Patienten mit einem oder mehreren der folgenden Alarmsymptome, Dysphagie, rezidivierendes Erbrechen, Inappetenz, unklarer Gewichtsverlust, gastrointestinale Blutung und/oder unklare Eisenmangelanämie sollen zu einer frühzeitigen ÖGD-Endoskopie mit Entnahme von Biopsien überwiesen werden.
Die Diagnostik heute ist mit Zoom und Farbe besser geworden. Dennoch soll bei der histologischen Diagnose jeder intraepithelialen Neoplasie der Prozess einer kompetenten (dokumentierten) pathologischen Zweitmeinung im Sinne eines Vier-Augen-Prinzips durchgeführt werden. Da die Staging-Laparoskopie die Therapieentscheidungen beim lokal fortgeschrittenen Magenkarzinom verbessert, sollte sie vor Beginn der neoadjuvanten Therapie durchgeführt werden.

\section{Neue Empfehlungen zur perioperativen Versorgung}

Kliniken mit hoher Fallzahl haben eine geringere perioperative Letalität als Kliniken mit niedriger Fallzahl. Patienten sollte daher die Überweisung an Kliniken mit hoher Fallzahl angeboten werden.

Bezüglich der präoperativen bzw. neoadjuvanten Therapie soll bei lokalisiertem Magenkarzinom der Kategorien cT3 und resektablem cT4a eine perioperative Chemotherapie durchgeführt werden. Beim nicht-fernmetastasierten Adenokarzinom des ösophagogastralen Übergangs der Kategorien cT3 und resektablen cT4 Tumoren soll eine neoadjuvante Radiochemotherapie oder eine perioperative Chemotherapie erfolgen. Eine präoperative Radiochemotherapie soll außerhalb von Studien beim Magenkarzinom weiterhin nicht durchgeführt werden. Ein Re-Staging zur Bewertung des Ansprechens soll mit CT und ÖGD nach Abschluss der präoperativen Therapie erfol- gen. Bei dem seltenen Nachweis eines Tumorprogresses soll die Entscheidung über die weitere Therapie interdisziplinär durchgeführt werden. Nach präoperativer Therapie soll eine histologische Bestimmung des Tumorregressionsgrades nach Becker erfolgen.

In der Palliativsituation sollte bei asymptomatischen, nicht-blutenden Patienten eine Resektion des Primärtumors nicht durchgeführt werden. Nach R1-Resektion und nach makroskopisch inkompletter Resektion ohne Nachweis von Fernmetastasen soll zunächst die Möglichkeit einer kurativen Nachresektion geprüft werden. Falls diese nicht möglich ist, kann eine postoperative Radiochemotherapie nach Konsens in der interdisziplinären Tumorkonferenz erfolgen. Bei funktioneller Inoperabilität eines Patienten oder Irresektabilität eines lokal begrenzten Adenokarziom des Magens oder ösophagogastralen Übergangs kann eine definitive Radiochemotherapie durchgeführt werden.

\section{Systemische Therapie des Magenkarzinoms}

Bezüglich der medikamentösen Tumortherapie soll in der Palliativsituation in der Erstlinientherapie eine Platin-/Fluoropyrimidin-haltige Kombinationstherapie durchgeführt werden. Bei Vorliegen von Kontraindikationen gegen Platin kann alternativ Irinotecan/Fluoropyrimidin angewendet werden. Dabei handelt es sich um einen Off-Label-Use.

Während der Chemotherapie sollen das allgemeine Befinden des Patienten, Tumorsymptome und vitale Körperfunktionen regelmäßig geprüft werden. Unter laufender palliativer Chemotherapie sollte alle sechs bis zwölf Wochen eine klinische Re-Evaluation und geeignete Bildgebung erfolgen, um negative Entwicklungen der Erkrankung rechtzeitig zu erkennen und Patienten nicht unnötig lange unwirksamen Therapien auszusetzen bzw. die Chance auf ggfs. wirksamere Therapien zu ermöglichen.

Eine Zweitlinientherapie ist nun der Therapiestandard. Sie sollte Irinotecan, Docetaxel, 
Paclitaxel und Ramucirumab oder Paclitaxel und Ramucirumab beinhalten, wobei der Zulassungsstatus zu berücksichtigen ist.

Bei limitierter Metastasierung sollte eine Resektion von Primärtumor und Metastasen außerhalb von Studien nicht erfolgen. Im Einzelfall können erst intraoperativ entdeckte, limitierte Metastasen, wenn R0 resektabel, reseziert werden. Patienten mit synchron limitierten Metastasen sollte die Überweisung in eine Klinik mit hoher Fallzahl angeboten werden.

\section{Empfehlungen zur Ernäh- rungstherapie}

Bezüglich der Ernährung empfehlen die S3-Leitlinien, dass Patienten eine Ernährungstherapie erhalten sollen, wenn der Ernährungszustand so weit kompromittiert ist, dass ein hohes Risiko für Komplikationen besteht oder eine geplante onkologische Therapie deshalb nicht durchgeführt werden kann. Der Ernährungsstatus soll bei allen Patienten, beginnend mit der Diagnosestellung, bei jeder stationären Aufnahme und ambulantem Patien- tenkontakt beurteilt werden, um Interventionen frühzeitig einleiten zu können.

Zur Begleitung während einer multimodalen Therapie und zur Vorbereitung auf die funktionellen Auswirkungen einer Ösophagektomie oder Gastrektomie sollte bereits präoperativ eine Ernährungsfachkraft hinzugezogen werden. Patienten sollen auch ohne Zeichen einer Mangelernährung präoperativ für 5-7 Tage bilanzierte Trinklösungen einnehmen.

Dr. Ine Schmale, Westerburg

Quelle: Deutscher Krebskongress (DKK) 2018 vom 21. bis 23. Februar 2018, Berlin. 